\title{
Meteoroid stream of 12P/Pons-Brooks, December $\kappa$-Draconids, and Northern June Aquilids
}

\author{
D. Tomko and L. Neslušan
}

\author{
Astronomical Institute, Slovak Academy of Science, 05960 Tatranská Lomnica, Slovakia \\ e-mail: [dtomko; ne]@ta3.sk
}

Received 29 February 2016 / Accepted 10 May 2016

\begin{abstract}
Context. It was found that some parent bodies of meteoroid streams can be related to more than one meteor shower observable in the atmosphere of Earth. The orbits of the members of such showers must evolve to the locations, which are far from the orbit of their parent, to cross the orbit of the Earth. An extensive simulation of the stream evolution is necessary to reveal such a complex of showers of the given parent body.

Aims. We continue the investigation of the evolution of the theoretical stream originating from the comet 12P/Pons-Brooks to understand its meteor-shower complex in more detail.

Methods. We model a theoretical comet stream assuming an ejection of 10000 particles, representing the meteoroids, from its nucleus in several past perihelion passages. Adding to our previous work, here we also consider the Poynting-Robertson drag in our study of the particles' dynamics. The orbits currently occurring in a vicinity of the Earth's orbit are used to predict the showers associated with comet $12 \mathrm{P}$.

Results. Two nighttime and two daytime showers are predicted to originate from 12P. The showers must consist of only relatively large particles, which are influenced to only a small extent by the Poynting-Robertson drag, because in this case, it deflects the particles from the collisional course with the Earth when efficient. The shower predicted to have the most particles is the nighttime shower, which can clearly be identified to the December $\kappa$-Draconids, No. 336 in the IAU MDC list. Another predicted nighttime shower has no counterpart in the considered observational data. Some characteristics of this shower are vaguely similar to those of Northern June Aquilids, No. 164. The observed counterparts of two predicted daytime showers were not found in the observational data we used or in the IAU MDC list.
\end{abstract}

Key words. comets: individual: 12P/Pons-Brooks - meteorites, meteors, meteoroids

\section{Introduction}

Many small bodies, especially comet nuclei, move around the Sun in an eccentric orbit. The delivery of heat on their surface is changed and this circumstance causes a gradual disintegration of the surface. If the heating is large enough, a lot of gas escapes from some active areas. This gas drifts the dusty particles above the surface of which a large part escapes to the interplanetary space as meteoroids.

A short time after the escape, the meteoroids move around the Sun in the orbits which are similar to that of their parent body. The similarity concerns not only the shape of orbit, but also its orientation in space: the orbits are situated in a common corridor. The meteoroids moving in these orbits represent a meteoroid stream. It was found that, typically, the dispersion of the time of perihelion passage of individual stream meteoroids is fastest of all orbital elements, and so the meteoroids gradually disperse along the whole parent orbit, but remain in the common corridor for a relatively long time.

If the corridor is situated so it crosses the Earth's orbit, part of the meteoroid stream collides with our planet and we observe these collisions as a meteor shower.

With time, the corridor spreads to such a large volume that the meteoroids at its border can be no longer identified as part of the stream. It was found (e.g., Neslušan 1999; Kaňuchová \& Neslušan 2007; Tomko \& Neslušan 2012; Neslušan et al. 2013a,b; Neslušan \& Hajduková 2014; Jakubík \& Neslušan 2015) that this dispersion of stream may also happen in a systematic way. Planetary perturbations can deflect the meteoroids from the original corridor around the orbit of the parent to one or more other corridors. Within these corridors, the meteoroids can approach the Earth's orbit and collide with this planet in several filaments. These filaments then correspond to several observable meteor showers originating from a single parent body.

A case can occur where the original corridor is situated far from the Earth's orbit, but another corridor or several corridors which have evolved, cross it. In such a case, the corresponding meteor shower or showers cannot be predicted from the vicinity of the parent body orbit and that of Earth. The showers can be predicted only by modeling the stream and following its dynamical evolution in interplanetary space accounting for all relevant, especially planetary, perturbations.

Modeling theoretical streams, mainly those related to a specified parent body in a well-known orbit, began when computational techniques had progressed enough to enable this way of study of meteoroid streams. One of the first studies of this type was that performed by Williams et al. (1979). They studied the dynamical evolution of Quadrantids, which were represented by ten test particles. These particles were numerically integrated over the period 300 B.C. to A.D. 3780.

To confirm the relationship between the Geminids and asteroid 3200 Phaethon, Hunt et al. (1985) created the model of 20 test particles and integrated them over 5000 years. The authors found that both Geminids and Phaethon are in a very 
interesting orbital phase and will soon approach to the orbits of Earth and Venus. Babadzhanov \& Obrubov (1987) integrated eight test particles, representing the Quadrantids, for four millennia. McIntosh \& Jones (1988) performed a numerical modeling of the Halley comet meteoroid stream. They numerically integrated the orbital motion of up to 500 test particles over several millennia.

The above mentioned studies are only the first few examples. Later, the number of test particles and integration period gradually increased. It is beyond the scope of this paper to give a complete review of all relevant studies. We could describe here those by Wu \& Williams (1992, 1993), Williams et al. (2004b,a), Jones et al. (2006), Porubčan et al. (2006), Babadzhanov et al. (2008), Jenniskens \& Vaubaillon (2008), Wiegert et al. (2009), Christou \& Vaubaillon (2010), Williams \& Ryabova (2011), Babadzhanov et al. (2013), and other authors. A review of the techniques used for the modeling of meteoroid streams in the solar system was recently given by Vaubaillon et al. (2015).

The method we use in our study was first suggested and used by Neslušan (1999) who modeled the stream of periodic comet $14 \mathrm{P} /$ Wolf and found a relation between this stream and the $\alpha$-Capricornids. At the same time, a relationship between this comet and the disappeared comet D/1892 T1 was found. However it seems that the meteoroids released from 14P/Wolf could become the members of the $\alpha$-Capricornid stream only for a limited period, during their transit between the various corridors. Jenniskens \& Vaubaillon (2010) later found a more suitable parent body for the $\alpha$-Capricornids: asteroid 2002 EX12, which was also named as comet 169P/NEAT.

The question whether the parent body of the Quadrantids is comet 96P or asteroid 2003 EH1 was re-opened by Kaňuchová \& Neslušan (2007) who modeled the streams of both objects, using the same method as Neslušan (1999), and found a certain preference that the comet is a more suitable parent than the asteroid. The same approach was used to study and compare the meteoroid complexes of both the parents by Neslušan et al. (2013a,b). It was found that these objects, currently moving in two clearly different osculating orbits, can associate practically identical meteoroid complexes. There are several corridors and the meteoroids can approach the Earth's orbit in six filaments. Not only the Quadrantids, but also Northern and Southern $\delta$-Aquarids and daytime Arietids belong to these complexes. Two other showers were predicted. The first is the southern Arietids, which actually seem to be present in the CMOR radar data. The second is hypothetical, at the moment, a shower with the mean radiant in a vicinity of the south pole.

The brief review of the type of modeling we use here can be terminated with a mention of simulation of the streams of comet C/1917 F1 (Mellish) by Neslušan \& Hajduková (2014) and asteroid 3200 Phaethon by Jakubík \& Neslušan (2015). Comet Mellish was confirmed to be the parent body of December Monocerotids and, possibly, April $\rho$-Cygnids. Phaethon is confirmed, as expected, the parent body of Geminids, but most probably also of the daytime Sextantids.

In this work, we study the meteoroid stream, which is assumed to originate from the nucleus of comet 12P/Pons-Brooks. In accordance with the last above-mentioned studies, we model this comet's theoretical stream and follow its dynamical evolution in course to identify all observable meteor showers from it. The method used is briefly reviewed in Sect. 2. The past evolution of the parent-body orbit, over the last 35.5 millennia, is analyzed in Sect. 3. The result - the prediction of potential showers, and the attempt to identify them to the corresponding real showers is described in Sect. 4. In the short Sect. 5, we deal with the ecliptic-toroidal structure found in the predicted filaments of the stream. The concluding remarks are presented in the last, sixth section.

\section{Stream modeling}

The method used to study of the orbital evolution of the modeled theoretical stream associated with a given parent body was suggested by Neslušan (1999). The procedure was recently described in a more detail in the paper by Tomko \& Neslušan (2012). It consists of several steps.

First we integrate the catalog orbit of the parent comet, $12 \mathrm{P} /$ Pons-Brooks, backward in time for a chosen period, $t_{\mathrm{ev}}$, which is regarded as the evolutionary period of future stream (see the third step below). The integration is performed using integrator RA15 created by Everhart (1985), which is the part of the MERCURY package (Chambers 1999). The gravitational perturbations of eight planets, from Mercury to Neptune, are considered in the integration. The backward integration is terminated exactly at the time of the comet's perihelion passage which is the closest to the assumed period. The non-gravitational effects are not considered during this integration.

In the second step, we model a theoretical stream at the moment of the perihelion passage of comet, reached in the previous integration. Specifically, we consider 10000 particles that are assumed to be randomly ejected in all directions from the surface of the parent body. In agreement with Sykes et al. (1989) and Neslušan (1999), we choose the size of the ejection velocity equal to $0.001 v_{\mathrm{p}}$, where $v_{\mathrm{p}}$ is the magnitude of the orbital velocity of parent body in the perihelion.

Of course, the real stream meteoroids are released from the surface of the parent body not only in its perihelion and with the uniform speed. Neither is their directional distribution typically random. In our model, however, we aim to fill in the appropriate phase space of orbital elements with the meteoroid particles. After a relaxation time, there is no significant difference between our simple model and a model created in a more sophisticated way.

We can suppose that the real meteoroids are released from the comet nucleus at every perihelion passage (or many perihelion passages). Therefore, we repeat the modeling of the stream and its follow up for several evolutionary periods. Specifically, we consider $t_{\mathrm{ev}}=50,100,250,350$, and 500 orbital revolutions of the parent body in the nominal orbit, $P_{\mathrm{o}}$. In the perihelia corresponding with these times before the present, the size of the ejection velocity, as $0.001 v_{\mathrm{p}}$, is equal to $37.58,47.14,37.51$, 34.99 , and $35.26 \mathrm{~m} \mathrm{~s}^{-1}$, respectively.

In the third step, we use integrator RA15 (Everhart 1985) to integrate all modeled particles from the past until the present. Also in this case, as in the integration of the comet orbit, the gravitational perturbations of eight planets are considered. The final characteristics of eight planets in the integration of the comet orbit in the second step are taken as initial in the integration of the particles from the past to the present.

In the first series of our models, we consider only the gravitational planetary perturbations. However, we know that the meteoroid particles are also influenced by the non-gravitational effects, especially by the solar radiation causing the so-called Poynting-Robertson (P-R) drag. This non-gravitational force is significant especially on the long time scale. Thus, we also repeat our modeling in the cases of $t_{\mathrm{ev}}=500$ and $350 P_{\mathrm{o}}$ for several values of the strength of the P-R drag, which is specified with parameter $\beta$, that is with the ratio of the magnitudes of both 
drag and solar gravitational forces. The radial component of the acceleration due to the P-R drag is $a_{r}=\beta\left(G M_{\odot} / r^{2}\right)\left(1-2 v_{r} / c\right)$, the transversal component equals $a_{t}=-\beta\left(G M_{\odot} / r^{2}\right) v_{t} / c$, and the perpendicular component is supposed to be zero. We denoted: $G$ - gravitational constant, $M_{\odot}-$ mass of the Sun, $v_{r}$ and $v_{t}-$ radial and transversal components (both in the orbital plane) of the particle's velocity in given moment, $c$ - speed of light, and $r$ - heliocentric radial distance of the particle.

We note that the influence of the P-R drag has not been often considered in the dynamical studies of meteoroid stream. Of the works mentioned in Sect. 1, the P-R effect was taken into account only by McIntosh \& Jones (1988) and in the works coauthored by Vaubaillon (Jenniskens \& Vaubaillon 2008; Wiegert et al. 2009; Christou \& Vaubaillon 2010), in which the method of sofisticated stream modeling (Vaubaillon 2004; Vaubaillon et al. 2005) was used. Recently, the P-R drag was considered by Jakubík \& Neslušan (2015) in their study of the Phaethon's meteoroid stream.

When the integration of the particle orbits is completed, we select, in the fourth step, the particles in the orbits passing around the Earth's orbit at the distance shorter than 0.05 au. The dynamical characteristics of these particles are similar to those that really collide with the Earth and, if they are numerous enough, they are used to predict a meteor shower.

In the last step, we attempt to identify the showers predicted on the basis of the particles approaching the Earth's orbit with the meteor showers actually observed. For this purpose, we use four available databases: the photographic IAU MDC (Lindblad et al. 2003), radio-meteor (Hawkins 1963; Sekanina \& Southworth 1975; Lindblad 2003), SonotaCo videometeor (SonotaCo 2009), and EDMOND video-meteor (Kornoš et al. 2014) databases. The identification and selection is done using the break-point method suggested by Neslušan et al. $(1995,2013 c)$. For a given threshold value of the Southworth \& Hawkins (1963) $D$-discriminant, $D_{\text {lim }}$, all meteors in the orbits with $D \leq D_{\text {lim }}$ are selected, where $D$ is the value of the discriminant between the orbit of an individual meteor from database and an actual mean orbit of the predicted shower. The mean orbit is calculated by averaging the orbits of particles approaching the Earth's orbit within 0.05 au.

\section{On the orbital evolution of parent comet}

The longest evolutionary period of stream that we study is about 35.5 millennia. The stability of the parent comet orbit during this period is a separate problem, which we investigate before the proper stream modeling. The heliocentric ecliptic orbital elements of $12 \mathrm{P}$ referred to the equinox J2000.0 are $q=0.773667 \mathrm{au}, a=17.121223 \mathrm{au}, e=0.954812$, $\Omega=255.891144^{\circ}, \omega=199.028469^{\circ}, i=74.176894^{\circ}$, and $T=2434885.380850$ for epoch $2435000.5^{1}$. To follow the past evolution of the comet in this orbit, including the uncertainity of its determination, we create 100 clones using the method by Chernitsov et al. (1998).

These authors derived that the orbit of $j$ th clone, written in the form of covariant $6 \times 1$ matrix $y_{j}$, can be calculated as

$y_{j}=y_{\mathrm{o}}+\mathbf{A} \eta^{\mathbf{T}}$,

where $y_{\mathrm{o}}$ is covariant $6 \times 1$ matrix with the nominal orbital elements, $\mathbf{A}$ is a triangle matrix such that the product $\mathbf{A} \mathbf{A}^{T}$ equals the covariance matrix related to the process of nominal orbit determinantion (the covariance matrix is given together with the

\footnotetext{
http://ssd.jpl.nasa.gov/sbdb.cgi
}

orbital elements on the JPL-browser web pages). Since we consider six orbital elements, $\mathbf{A}$ is a $6 \times 6$ matrix. $\eta$ is a contravariant $1 \times 6$ matrix. Each of its elements is a random value from the interval $(0,1)$. Its covariant form is $\eta^{T}$.

The orbits of clones and nominal orbit were integrated backward in time for the above-mentioned period of $~ 35500$ years. The resultant evolution of the individual elements is shown in Fig. 1. We see that the orbit of $12 \mathrm{P}$ was practically identical to the orbits of all clones in the last six millennia. So, there is a large probability that the nominal comet orbit was its real orbit during this period.

In the period from about 6 to $35.5 \mathrm{kyr}$ ago, a given element of comet orbit is often shifted, from the center of the dispersion of this element of clones, to the lower or upper border of the dispersion interval. Nevertheless, each element is always well inside this interval and we can conclude that the nominal comet orbit is not any extremal orbit in this respect. Therefore, we use it as the reference orbit in our modeling of stream. (It would be, anyway, hard to find an orbit that has all its elements in the center of the dispersion interval during the whole period considered.)

We also see in Fig. 1 that the perihelion distance (plot a), eccentricity (c), and inclination (f) are coupled. This indicates that the motion of comet is controlled by a planet, most probably by Jupiter.

Since the evolution of the 12P's elements is not permanently smooth, we also search for the close encounters of the comet with the perturbing planets. During the considered maximum evolutionary period, the comet approached the Earth (and no other planet), within the limit of $0.1 \mathrm{au}$, five times. The closest approach happened 19937 years before the present to the minimum distance about $0.04 \mathrm{au}$, which is an order of magnitude larger than the Earth-Moon distance. The comet orbit was not significantly influenced by the Earth's gravity even at this event.

Within the limit of $0.5 \mathrm{au}$, there were several approaches to Venus, Earth, and Mars (as well as a single approach to Mercury to the distance of $0.49 \mathrm{au}$ ). In addition, the comet approached six times within the 0.5 -au limit to Saturn. These approaches happened $9084,9349,14474,14621,17390$, and 21537 years before the present at distances of about $0.21,0.49,0.36,0.17$, 0.43 , and 0.27 au, respectively. As we see in Fig. 1 the times of the approaches do not correspond to those of sudden changes of 12P's orbit. Obviously, the perturbations by Saturn have not been very significant, either.

Some sudden changes of the $12 \mathrm{P}$-orbit, seen as a steep increase or decrease of the value of an element (or elements) in the plots of Fig. 1, are likely the consequences of distant planetary resonance action. Actually, we performed one more test of the orbital stability of $12 \mathrm{P}$ : we integrated its orbit backward eight times, for the periods of $5,10,15,29,25,26,27$, and 30 millennia. Then, each integration was turned to proceed forward, until the starting time of the first integration was reached. The final and starting orbits should be identical if no significant perturbation, leading to a chaotic behavior of the orbit, occurred during the investigated period.

The integrations for the periods of 5 to 25 millennia provided such a result. However those for the periods longer than 25 millennia resulted in a final orbit that was different from the starting orbit. A sudden jump occurred in the values corresponding elements when we compared them for (i) 25 millennia and present day and (ii) 26 or more millennia and present day. For example, for the period of 26 millennia, the differences between the starting and final perihelion distance, eccentricity, argument of perihelion, longitude of ascending node, and inclination were $0.16 \mathrm{au},-0.012,-13.7^{\mathrm{o}}, 12.8^{\circ}$, and $3.4^{\mathrm{o}}$, respectively. 

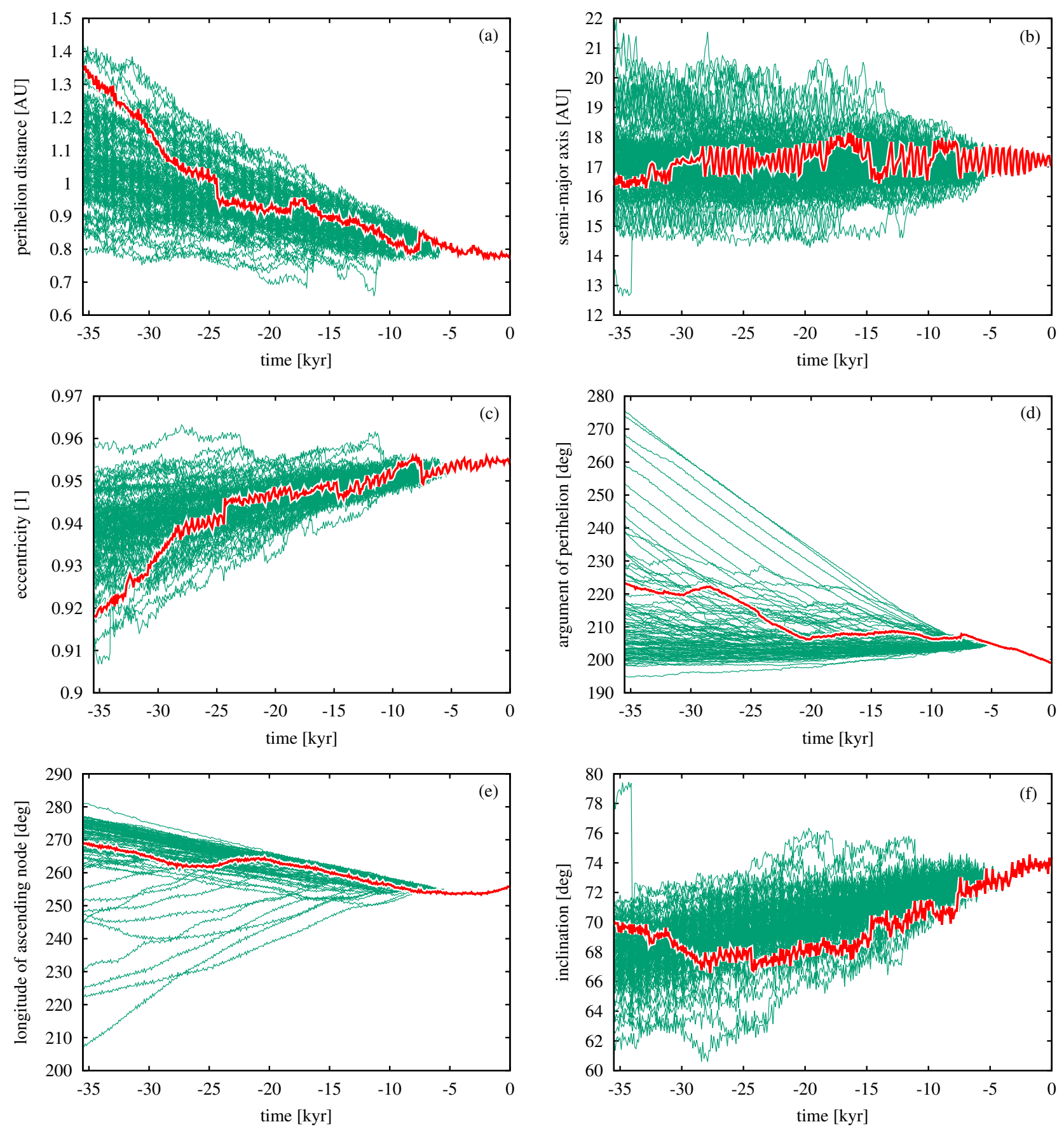

Fig. 1. Evolution of the perihelion distance (plot a)); semi-major axis b); eccentricity c); argument of perihelion d); longitude of ascending node e); and inclination to the ecliptic f) of the orbit of comet 12P/Pons-Brooks (thick red curve) over the past 35.5 millennia. The corresponding evolution of the orbital elements of 100 cloned orbits (dashed green curves; see Sect. 3) is also shown.

Hence, our last model for $t_{\mathrm{ev}}=500 P_{\mathrm{o}}$ (35422 years) is highly uncertain and we perform this modeling to see a wider context: if our statistical-type study can predict a reasonable shower, similar to those from other models, also beyond the limit of the chaos.

\section{Prediction of showers from the modeled streams}

In a prediction of a meteor shower observable in the Earth's atmosphere, it is worthwhile to know the location of the potential parent body's orbit in space in respect to the Earth's orbit. In Fig. 2, we show the minimum distance between the orbits of both comet 12P and our planet during the last 25 millennia, that is about 350 orbital revolutions of the comet around the Sun in its nominal orbit. The minimum distance of the comet's preperihelion and post-perihelion orbital arcs is shown separately. The pre-perihelion arc was within 0.2 au of the Earth's orbit for the whole of the investigated period. In the period from about 14 to $27 \mathrm{kyr}$ ago, the orbit of $12 \mathrm{P}$ almost crossed, several times, the orbit of our planet.

If a meteoroid stream forms around the comet orbit, a similar evolution of the orbits of many individual meteoroids can be expected, at least during a limited period after the particles were released from the parent. Assuming a certain dispersion of the 


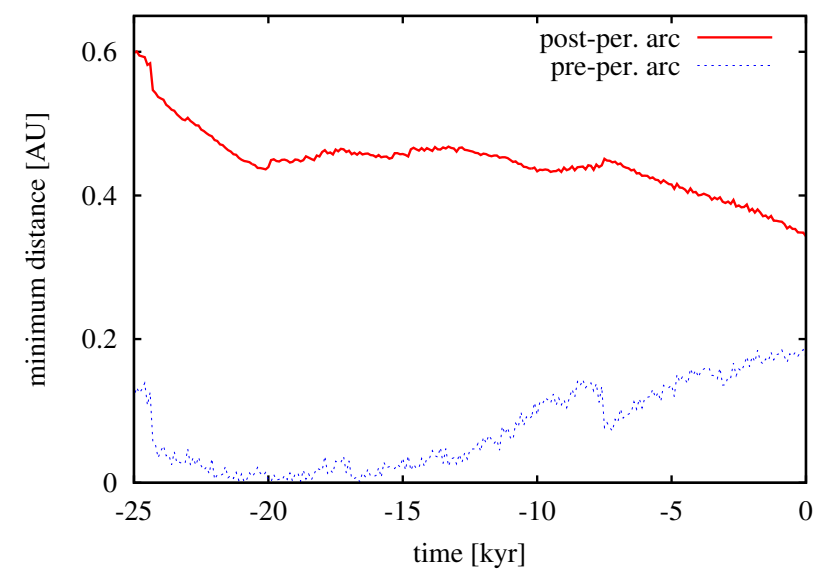

Fig. 2. Evolution of the minimum distance between the orbits of both Earth and comet 12P/Pons-Brooks. The minimum distance of the postperihelion (red solid curve) and pre-perihelion (blue dashed curve) arcs of the comet to the Earth's orbit is shown separately.

orbits of these particles, many of their orbits obviously approach the Earth's orbit also at the present and the particles can collide with this planet.

To map the dynamics of the meteoroid particles released from the parent in various times (around various perihelion passages), we model the theoretical stream for several evolutionary times. Specifically, this is done for $t_{\mathrm{ev}}=50,100,250,350$, and $500 P_{\mathrm{o}}(3542,7084,17711,24795$, and 35422 years $)$. We recall here that $P_{\mathrm{o}}$ is the orbital period of $12 \mathrm{P}$ in its nominal orbit $\left(P_{\mathrm{o}}=70.843\right.$ years $)$.

In the first series of models, the P-R drag is ignored. To gain a picture of the evolution of a theoretical stream, we construct the evolution of distribution of orbital elements of at least one modeled stream, that for the evolutionary time $t_{\mathrm{ev}}=350 P_{\mathrm{o}}$. As discussed in Sect. 3, it is the maximum time during which the nominal orbit of $12 \mathrm{P}$ can be reliably traced backward. The distribution is demonstrated in Fig. 3. As expected, the stream develops from the initially compact configuration into a less compact one. Our way of modeling yields the distribution of every orbital element around a single peak. With time, this peak becomes wider and small secondary peaks also occur. These peaks can be well distinguished in the distributions of perihelion distance and eccentricity.

It appeared that it is at the present $(0 \mathrm{kyr})$ that the largest number of modeled particles, representing the meteoroids, approached the Earth's orbit in the model for evolutionary time $t_{\mathrm{ev}}=500 P_{\mathrm{o}}$. The distribution of the radiants of these particles is shown in Fig. 4a. We see that the stream approaches the Earth's orbit in four separated filaments, which are labeled as F1, F2, F3, and F4.

In Sect. 3, we concluded that the evolution of the nominal orbit of the parent comet to a deeper past than 25 millennia cannot be followed reliably and, consequently, the model for $t_{\mathrm{ev}}=500 P_{\mathrm{o}}$ does not provide any reliable prediction. We carry out this model in order to map a wider context. Therefore, we further discuss, mainly, the result yielded from the model for $t_{\mathrm{ev}}=350 P_{\mathrm{o}}$, which does not, however, predict filament F4. The distribution of radiants of the particles in this model, which approach the Earth's orbit within $0.05 \mathrm{au}$, is shown in Fig. 5a.

The most numerous filament is F1. It is predicted by almost all models. Less numerous filaments F2 and F3 do not occur for $t_{\mathrm{ev}}=250 P_{\mathrm{o}}$ and both consist of only a single meteor for $t_{\mathrm{ev}}=100 P_{\mathrm{o}}$. No filament, even the most abundant $\mathrm{F} 1$, is predicted for the shortest period $t_{\mathrm{ev}}=50 P_{\mathrm{o}}$. Filament F4 is predicted only in the uncertain model for $t_{\mathrm{ev}}=500 P_{\mathrm{o}}$, in which comprises few meteors. Nevertheless, this prediction is important in respect to the ecliptic-toroidal structure discussed in Sect. 5 .

Since there is a prediction of regular showers, more than a single, only for $t_{\mathrm{ev}}=500$ and $350 P_{\mathrm{o}}$, we consider the further models, in which the motion of meteoroids is influenced by the P-R drag, only for these two evolutionary periods. Specifically, we consider the models with the values of parameter $\beta$ equal to $0.001,0.005$, and 0.009 . The distributions of the radiants of particles approaching the Earth's orbit within 0.05 au for all these models are shown in Fig. 4 (for the models with $t_{\mathrm{ev}}=500 P_{\mathrm{o}}$ ) and Fig. 5 (for those with $t_{\mathrm{ev}}=350 P_{\mathrm{o}}$ ).

The mean orbital characteristics of all predicted showers, corresponding to filaments F1 to F4, are given in Table 1 and corresponding geophysical parameters are given in Table 2 . We also tried to separate the real showers corresponding to all filaments from the four databases used (listed in Sect. 2). No shower was separated from the photographic IAU MDC and radio-meteor databases. This means that no concentration of the meteor orbits in the orbital phase space, indicated by our prediction, could be distinguished from the sporadic background, in these databases. The characteristics of the showers successfully separated from the SonotaCo video data are also listed in Tables 1 and 2.

To use the break-point method, we need an initial orbit to start an iteration procedure. Naturally, the mean orbit of a given predicted shower is the most appropriate initial orbit. Since there are the predictions for more than a single time $t_{\mathrm{ev}}$, we have more than only a single initial orbit for each shower. We do the separation for every initial orbit and, consequently, there are two (or three) separated orbits for a single filament in Tables 1 and 2. The initial orbit used is indicated with the values of $t_{\mathrm{ev}}$ and $\beta$ in the parentheses, in the first two columns of the tables.

In the case of $12 \mathrm{P}$-stream, the action of the P-R drag tends to deflect the meteoroid from the collisional course with our planet. In the models for $t_{\mathrm{ev}}=350 P_{\mathrm{o}}$ with the P-R drag considered, only the most abundant filament F1 is predicted (Fig. 5). Its numerousity decreases with increasing value of parameter $\beta$. Concerning the other filaments, F2 consists of only a single particle in the model with $\beta=0.001$. Filaments F3 and F4 do not occur even for this low $\beta$-value and no filament, except of $\mathrm{F} 1$, is predicted for the considered $\beta=0.005$ or $\beta=0.009$.

Athough the nominal orbit is uncertain in the time more than about 25 millennia ago, the prediction of the filaments in models for $t_{\mathrm{ev}}=500 P_{\mathrm{o}}$ is consistent with the trend set by the models for a shorter $t_{\mathrm{ev}}$. We can see this in, for example, the distribution of radiants shown in Fig. 4. All four filaments exist only when the $\mathrm{P}-\mathrm{R}$ drag is negligible (Fig. $4 \mathrm{a}$ for $\beta=0$ ). For $\beta=0.001$, filament F2 is rarefied, F3 consists of only two meteors, and F4 of only a single meteor. Another meteor has its radiant largely shifted toward the equator and it cannot be regarded as a member of F4.

For an even stronger P-R drag, with $\beta=0.005$, filaments F3 and F4 disappear, and F2 consists of only a single meteor. In more detail, there are two radiants near the radiant area of F4, but largely shifted toward the equator. For $\beta=0.009$, the stream approaches the Earth's orbit only in the single filament, F1.

Some further properties of individual filaments in the models and their correspondence to real showers, if they exist, are described in the following sub-sections. 
Table 1. Mean orbital characteristics of the predicted meteor showers associated with comet 12P/Pons-Brooks.

\begin{tabular}{|c|c|c|c|c|c|c|c|c|}
\hline$t_{\mathrm{ev}}\left[P_{\mathrm{o}}\right]$ & $\beta$ & $q[a u]$ & $a[a u]$ & $e$ & $\omega\left[{ }^{\circ}\right]$ & $\Omega\left[{ }^{\circ}\right]$ & $i\left[^{\mathrm{o}}\right]$ & $N_{\text {sel. }}$ \\
\hline \multicolumn{9}{|c|}{ Filament F1 } \\
\hline \multicolumn{9}{|c|}{ Predicted by simulations in this work: } \\
\hline 100 & 0.0 & $0.911 \pm 0.066$ & $19.213 \pm 2.461$ & $0.952 \pm 0.007$ & $209.321 \pm 2.922$ & $247.213 \pm 3.013$ & \pm 5.113 & 195 \\
\hline 250 & 0.0 & $0.902 \pm 0.037$ & $17.698 \pm 1.871$ & $0.948 \pm 0.006$ & $213.811 \pm 5.906$ & $243.335 \pm 3.878$ & $74.040 \pm 2.367$ & 1227 \\
\hline \multirow[t]{3}{*}{350} & 0.0 & $0.901 \pm 0.036$ & $17.781 \pm 2.202$ & $0.949 \pm 0.006$ & $213.771 \pm 5.716$ & $242.831 \pm 3.920$ & $74.274 \pm 2.713$ & 1378 \\
\hline & 0.001 & $0.911 \pm 0.037$ & $17.696 \pm 1.961$ & $0.948 \pm 0.006$ & $210.218 \pm 6.735$ & $244.029 \pm 4.795$ & $74.039 \pm 3.001$ & 1037 \\
\hline & 0.005 & $0.951 \pm 0.033$ & $17.200 \pm 1.728$ & $0.944 \pm 0.006$ & $201.315 \pm 6.389$ & $246.749=$ & $76.467=$ & 824 \\
\hline \multirow[t]{4}{*}{500} & 0.0 & $0.911 \pm 0.042$ & $19.435 \pm 3.695$ & $0.951 \pm 0.009$ & $212.163 \pm 8.779$ & 245.939 & $72.399 \pm 4$ & 761 \\
\hline & 0.001 & $0.917 \pm 0.044$ & $19.348 \pm 3.848$ & $0.951 \pm 0.009$ & 208.334 & 41 & 19 & 624 \\
\hline & 0.005 & $0.959 \pm 0.033$ & $18.300 \pm$ & $0.947 \pm 0.007$ & $197.469=$ & 249.760 & 92 & 681 \\
\hline & 0.009 & $0.973 \pm 0.029$ & $18.020 \pm 1.742$ & $0.946 \pm 0.005$ & $195.734 \pm 4.242$ & $246.868 \pm 3$ & $76.966 \pm 2$ & 267 \\
\hline \multicolumn{9}{|c|}{ Separated from the SonotaCo video data in this work: } \\
\hline$(350)$ & $(0.0)$ & $0.925 \pm 0.009$ & $12.525 \pm 12.538$ & $0.905 \pm 0.044$ & 209.518 & 251.267 & 72. & 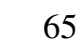 \\
\hline$(500)$ & $(0.0)$ & $0.924 \pm 0.006$ & $11.504 \pm 4.217$ & $0.911 \pm 0.028$ & 209.676 & 250.679 & $72.996 \pm 1$ & 40 \\
\hline \multicolumn{9}{|c|}{ Separated from the EDMOND video data in this work: } \\
\hline$(350)$ & $(0.0)$ & $0.928 \pm 0.010$ & $11.171 \pm 10.524$ & $0.885 \pm 0.046$ & 208. & 40 & 72 & 111 \\
\hline$(500)$ & $(0.0)$ & $0.928 \pm 0.008$ & $8.564 \pm 3.193$ & $0.879 \pm 0.038$ & $208.951=$ & 17 & 1.800 & 7 \\
\hline \multicolumn{9}{|c|}{ December $\kappa$-Draconids by Jenniskens et al. (2016): } \\
\hline & & 0.929 & 10.31 & 0.914 & 208.5 & 251.5 & 73.1 & 6 \\
\hline \multicolumn{9}{|c|}{ Filament F2 } \\
\hline \multicolumn{9}{|c|}{ Predicted by simulations in this work: } \\
\hline 100 & 0.0 & 0.067 & 15.914 & 0.996 & 212.946 & 220.715 & 25.850 & \\
\hline \multirow[t]{2}{*}{350} & 0.0 & $0.104 \pm 0.073$ & $13.337 \pm 5.108$ & $0.987 \pm 0.018$ & $208.509 \pm 6.095$ & $233.233 \pm 11.631$ & $72.296 \pm 59.256$ & \\
\hline & 0.001 & 0.059 & 12.981 & 0.995 & 207.581 & 220.695 & 51.312 & \\
\hline \multirow[t]{3}{*}{500} & 0.0 & $0.065 \pm 0.028$ & $14.579 \pm 3.173$ & $0.996 \pm 0.001$ & $208.017 \pm 6.374$ & $229.581 \pm 6.884$ & $51.213 \pm 14.299$ & 43 \\
\hline & 0.001 & $0.049 \pm 0.026$ & $13.206 \pm 2.821$ & $0.996 \pm 0.001$ & $204.560 \pm 6.777$ & $232.900 \pm 7.773$ & $56.426 \pm 18.503$ & 21 \\
\hline & 0.005 & 0.0308 & 10.031 & 0.997 & 201.878 & 231.076 & 46.643 & \\
\hline
\end{tabular}

Predicted by simulations in this work:

$\begin{array}{lllccccr}100 & 0.0 & 0.273 & 32.447 & 0.992 & 283.449 & 157.741 & 6.003\end{array}$

$\begin{array}{llllllll}350 & 0.0 & 0.141 \pm 0.126 & 18.933 \pm 5.504 & 0.993 \pm 0.003 & 317.238 \pm 20.110 & 104.531 \pm 30.164 & 39.461 \pm 13.314\end{array}$

$\begin{array}{lllllll}0.001 & 0.106 & 14.556 & 0.993 & 324.081 & 92.553 & 47.649\end{array}$

$\begin{array}{lllllllll}500 & 0.0 & 0.074 \pm 0.042 & 15.073 \pm 2.881 & 0.995 \pm 0.002 & 330.334 \pm 7.957 & 87.462 \pm 13.320 & 56.030 \pm 20.123 & 14\end{array}$

$\begin{array}{llllllll}0.001 & 0.037 \pm 0.000 & 9.191 \pm 1.828 & 0.996 \pm 0.000 & 338.236 \pm 1.292 & 79.523 \pm 3.015 & 60.469 \pm 8.508 & 2\end{array}$

Separated from the SonotaCo video data in this work:

$\begin{array}{llllll}(350) & (0.0) & 0.104+0.018 & 2.549+2.468 & 0.943 \pm 0.030 & 328.433\end{array}+3.640$

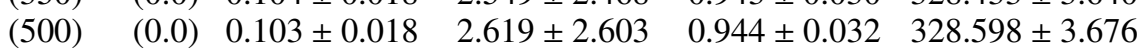

Separated from the EDMOND video data in this work:

$\begin{array}{llllll}(350) & (0.0) & 0.122 \pm 0.016 & 1.946 \pm 0.485 & 0.934 \pm 0.019 & 325.525+2.751\end{array}$

$\begin{array}{llllll}(500) & (0.0) & 0.121 \pm 0.023 & 1.897 \pm 0.561 & 0.930 \pm 0.027 & 326.061 \pm 3.749\end{array}$

$97.253 \pm 2.990 \quad 43.998 \pm 4.557$

19

$97.156 \pm 3.083 \quad 45.018 \pm 3.584 \quad 17$

$108.985 \pm 3.040 \quad 35.230 \pm 2.036 \quad 68$

$107.420 \pm 5.094 \quad 35.671 \pm 3.484 \quad 163$

Northern June Aquilids by Sekanina (1976):

$\begin{array}{lllllll}0.114 & 1.348 & 0.916 & 329.5 & 85.8 & 39.3 & 35\end{array}$

Northern June Aquilids by Brown et al. (2008):
0.11
1.71
0.936
327.7
101.3
39.4
1377

Northern June Aquilids by Holman \& Jenniskens (2012):

$0.124 \quad 2.11$

324.60

108.09

37.60

131

Northern June Aquilids by Jenniskens et al. (2016):

$\begin{array}{lllll}0.114 & 1.79 & 0.937 & 326.9 & 101.7\end{array}$

Filament F4

Predicted by simulations in this work:

\begin{tabular}{ccccccccc}
500 & 0.0 & $0.873 \pm 0.028$ & $14.904 \pm 4.101$ & $0.939 \pm 0.016$ & $316.810 \pm 4.187$ & $73.903 \pm 1.728$ & $80.853 \pm 680$ & 3 \\
& 0.001 & 0.815 & 12.546 & 0.935 & 311.768 & 70.476 & 80.299 & 1 \\
\hline
\end{tabular}

Notes. The parameters of the corresponding showers separated from the databases used as well as well-known corresponding showers found by other authors are also listed. Symbols used are as follows: $t_{\mathrm{ev}}$ - period of the following of orbital evolution (the theoretical stream was modeled before this time); $q$ - perihelion distance; $a$ - semi-major axis; $e$ - eccentricity; $\omega-$ argument of perihelion; $\Omega-$ longitude of ascending node; $i-$ inclination to the ecliptic; and $N_{\text {sel. }}$ - the number of particles or real meteors selected from the modeled set (of 10000 particles) or from the given database. Time $t_{\mathrm{ev}}$ is given in multiples of the nominal orbital period of 12P. The values of $t_{\mathrm{ev}}$ and $\beta$ in parentheses at the orbits separated from the SonotaCo database indicate the corresponding predicted orbit, which was considered as initial in the iteration procedure within the separation. 
D. Tomko and L. Neslušan: Meteoroid stream of 12P/Pons-Brooks

Table 2. Mean geophysical characteristics of the predicted meteor showers associated with comet 12P/Pons-Brooks.

\begin{tabular}{|c|c|c|c|c|c|c|c|}
\hline$t_{\mathrm{ev}}\left[P_{\mathrm{o}}\right]$ & $\beta$ & $\lambda_{\odot}\left[{ }^{\circ}\right]$ & $\alpha_{\mathrm{g}}\left[^{\circ}\right]$ & $\delta_{\mathrm{g}}\left[{ }^{\circ}\right]$ & $V_{\mathrm{g}}\left[\mathrm{km} \mathrm{s}^{-1}\right]$ & $V_{\mathrm{h}}\left[\mathrm{km} \mathrm{s}^{-1}\right]$ & $\gamma\left[^{0}\right]$ \\
\hline & & & & nent $\mathrm{l}$ & & & \\
\hline \multicolumn{8}{|c|}{ Predicted by simulations in this work: } \\
\hline 100 & 0.0 & $247.305 \pm 2.335$ & $181.992 \pm 5.182$ & $73.183 \pm 3.377$ & $43.784 \pm 1.729$ & $41.856 \pm 0.068$ & 103.4 \\
\hline 250 & 0.0 & $243.324 \pm 3.890$ & $168.930 \pm 11.831$ & $74.002 \pm 3.142$ & $44.556 \pm 1.021$ & $41.794 \pm 0.062$ & 105.3 \\
\hline \multirow[t]{4}{*}{350} & 0.0 & $242.817 \pm 3.935$ & $168.390 \pm 11.559$ & $74.061 \pm 3.191$ & $44.657 \pm 1.164$ & $41.793 \pm 0.074$ & 105.2 \\
\hline & 0.001 & $243.996 \pm 4.810$ & $174.661 \pm 12.352$ & $73.305 \pm 4.183$ & $44.516 \pm 1.304$ & $41.796 \pm 0.064$ & 103.8 \\
\hline & 0.005 & $246.739 \pm 4.696$ & $187.567 \pm 9.164$ & $69.045 \pm 4.118$ & $45.528 \pm 1.355$ & $41.787 \pm 0.063$ & 99.4 \\
\hline & 0.009 & $246.854 \pm 3.112$ & $193.185 \pm 5.709$ & $67.371 \pm 2.797$ & $45.747 \pm 1.113$ & $41.812 \pm 0.060$ & 96.7 \\
\hline \multirow[t]{4}{*}{500} & 0.0 & $245.956 \pm 6.497$ & $173.227 \pm 16.639$ & $73.778 \pm 5.470$ & $43.833 \pm 1.785$ & $41.844 \pm 0.102$ & 105.3 \\
\hline & 0.001 & $247.593 \pm 7.062$ & $179.397 \pm 16.949$ & $72.044 \pm 6.220$ & $44.164 \pm 1.835$ & $41.848 \pm 0.099$ & 103.6 \\
\hline & 0.005 & $249.761 \pm 5.928$ & $194.038 \pm 9.848$ & $67.433 \pm 5.391$ & $45.178 \pm 1.601$ & $41.830 \pm 0.077$ & 97.8 \\
\hline & 0.009 & $246.876 \pm 3.098$ & $193.235 \pm 5.662$ & $67.367 \pm 2.802$ & $45.741 \pm 1.110$ & $41.812 \pm 0.060$ & 96.7 \\
\hline \multicolumn{8}{|c|}{ Separated from the SonotaCo video data in this work: } \\
\hline$(350)$ & $(0.0)$ & $251.267 \pm 1.848$ & $185.580 \pm 3.807$ & $70.940 \pm 1.884$ & $43.479 \pm 1.077$ & $41.333 \pm 0.508$ & 103.0 \\
\hline$(500)$ & $(0.0)$ & $250.679 \pm 0.841$ & $184.635 \pm 2.159$ & $71.011 \pm 1.529$ & $43.723 \pm$ & $41.399 \pm$ & 103.0 \\
\hline \multicolumn{8}{|c|}{ Separated from the EDMOND video data in this work: } \\
\hline$(350)$ & $(0.0)$ & $251.661 \pm 1.840$ & $187.031 \pm 4.122$ & $70.393 \pm 1.740$ & $43.304 \pm 1.167$ & $41.109 \pm 0.541$ & 102.4 \\
\hline$(500)$ & $(0.0)$ & $251.495 \pm 1.046$ & $186.866 \pm 3.293$ & $70.549 \pm 1.368$ & $43.165=$ & $41.039 \pm$ & 102.5 \\
\hline \multicolumn{8}{|c|}{ December $\kappa$-Draconids by SonotaCo (2009) team: } \\
\hline & & 250.2 & 186.0 & 70.2 & 43.8 & - & - \\
\hline \multicolumn{8}{|c|}{ December $\kappa$-Draconids by Jenniskens et al. (2016): } \\
\hline & & 252.0 & 187.2 & 70.2 & 43.8 & - & - \\
\hline \multicolumn{8}{|c|}{ Filament F2 } \\
\hline \multicolumn{8}{|c|}{ Predicted by simulations in this work: } \\
\hline 100 & 0.0 & 43.922 & 17.583 & 0.518 & 46.119 & 42.223 & 28.255 \\
\hline \multirow[t]{2}{*}{350} & 0.0 & $57.681 \pm 23.391$ & $25.166 \pm 9.665$ & $1.692 \pm 8.318$ & $47.983 \pm 6.873$ & $40.675 \pm 0.952$ & 34.6 \\
\hline & 0.001 & 40.688 & 13.245 & -5.028 & 46. & 41. & 31.9 \\
\hline \multirow[t]{3}{*}{500} & 0.0 & $49.591 \pm 6.632$ & $21.456 \pm 3.962$ & $-1.195 \pm 2.414$ & $46.176 \pm$ & $41.010 \pm$ & 31.5 \\
\hline & 0.001 & $53.157 \pm 6.806$ & $23.087 \pm 4.032$ & $0.547 \pm 2.337$ & $47.233 \pm$ & $41.083 \pm 0.0 .837$ & 32.4 \\
\hline & 0.005 & 52.595 & 21.998 & 1.802 & 48.251 & 41.816 & 32.1 \\
\hline \multicolumn{8}{|c|}{ Filament F3 } \\
\hline \multicolumn{8}{|c|}{ Predicted by simulations in this work: } \\
\hline 100 & 0.0 & 143.484 & 336.501 & -6.050 & 36.005 & 41.531 & 166.9 \\
\hline \multirow[t]{2}{*}{350} & 0.0 & $103.208 \pm 27.680$ & $306.845 \pm 18.318$ & $-7.104 \pm 4.965$ & $43.291 \pm 5.376$ & $41.319 \pm 0.566$ & 153.3 \\
\hline & 0.001 & $93.319-$ & 300.138 & -7668 & 44.464 & 40.748 & 150.1 \\
\hline \multirow[t]{2}{*}{500} & 0.0 & $87.797 \pm 13.950$ & $297.649 \pm 10.078$ & $-10.162 \pm 4.066$ & $46.587 \pm 2.642$ & $40.953 \pm 0.760$ & 148.4 \\
\hline & 0.001 & $79.234 \pm 2.374$ & $292.092 \pm 1.182$ & $-13.459 \pm 0.225$ & $48.141 \pm 0.173$ & $40.780 \pm 0.767$ & 146.6 \\
\hline \multicolumn{8}{|c|}{ Separated from the SonotaCo video data in this work: } \\
\hline$(350)$ & $(0.0)$ & $97.253 \pm 2.990$ & $307.495 \pm 2.921$ & $-5.201 \pm 2.228$ & $39.609 \pm$ & $35.468 \pm$ & 146.2 \\
\hline$(500)$ & $(0.0)$ & $97.157 \pm 3.083$ & $307.610 \pm 3.076$ & $-4.982 \pm 2.256$ & $39.750 \pm 3.111$ & $35.462 \pm 3.267$ & 145.9 \\
\hline Separate & $\mathrm{d}$ from $\mathrm{t}$ & EDMOND video & ata in this work: & & & & \\
\hline$(350)$ & $(0.0)$ & $108.984 \pm 3.040$ & $316.286 \pm 2.298$ & $-3.620 \pm 1.634$ & $37.910 \pm$ & $35.508=$ & 149.0 \\
\hline$(500)$ & $(0.0)$ & $107.420 \pm 5.094$ & $315.220 \pm 3.902$ & $-3.739 \pm 2.417$ & $37.674 \pm 2.298$ & $35.142 \pm 2.106$ & 148.4 \\
\hline Norther & June A & uilids by Sekanina & 976): & & & & \\
\hline & & 86.0 & 298.3 & -7.1 & 36.3 & 32.5 & - \\
\hline Norther & June A & uilids by Brown et & (2008): & & & & \\
\hline & & 101.5 & 310.4 & -4.09 & 38.4 & - & - \\
\hline Norther & June A & uilids by Holman \& & Tenniskens (2012): & & & & \\
\hline & & 108.09 & 315.35 & -3.07 & 38.33 & - & - \\
\hline Norther & June A & uilids by Molau et a & (2012): & & & & \\
\hline & & 81.5 & 292.0 & -11.7 & 43.0 & - & - \\
\hline Norther & June A & uilids by Jennisken & et al. (2016): & & & & \\
\hline & & 101.0 & 309.7 & -5.3 & 38.3 & - & - \\
\hline & & & & nent F4 & & & \\
\hline Predict & by $\operatorname{sim}$ & lations in this work & & & & & \\
\hline 500 & 0.0 & 253.81 & 163.61 & -53.43 & 47.76 & 41.69 & 71.5 \\
\hline & 0.001 & 250.53 & 164.62 & -52.33 & 47.56 & 41.58 & 69.3 \\
\hline
\end{tabular}

Notes. The parameters of the corresponding showers separated from the databases used as well as well-known corresponding showers found by other authors are also listed. The symbols used are as follows: $t_{\mathrm{ev}}-$ as in Table $1 ; \lambda_{\odot}-$ solar longitude of assumed or observed maximum of the shower activity; $\alpha_{\mathrm{g}}$ and $\delta_{\mathrm{g}}-$ equatorial coordinates of geocentric radiant; $V_{\mathrm{g}}$ and $V_{\mathrm{h}}-$ geocentric and heliocentric velocity; and $\gamma-$ angular distance of the mean radiant from the Sun in time corresponding to the mean longitude. The values of $t_{\mathrm{ev}}$ and $\beta$ in the parentheses at the orbits separated from the SonotaCo database indicate the corresponding predicted orbit, which was considered as initial in the iteration procedure within the separation. 

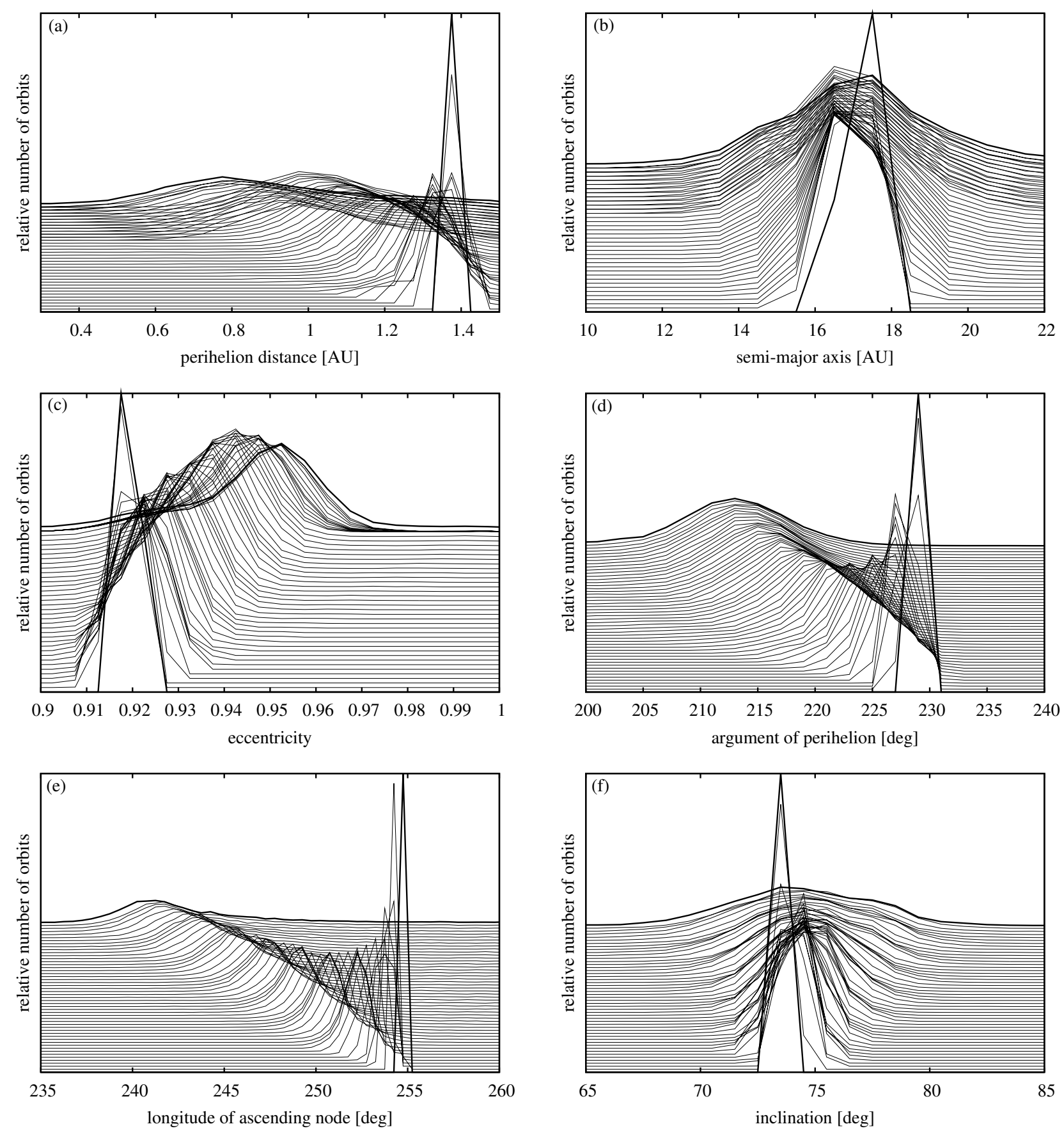

Fig. 3. Evolution of the orbital-element distributions of theoretical stream associated with comet 12P/Pons-Brooks. The stream is modeled in the time of 12P-perihelion passage, which happened before 350 nominal orbital periods of the comet. The bottom-based curve in each plot illustrates the distribution in the time of modeling. The curves with the base shifted vertically up about an equidistant section show the behavior for another successive 500-year intervals. The top-based curve shows the distribution at the present.

\subsection{Filament F1 - December $\kappa$-Draconids}

Filament F1 is the most abundant in each model by which it is predicted. If we consider $\beta=0$, it contains $0,195,1227,1378$, and 761 particles in the models for $t_{\mathrm{ev}}=50,100,250,350$, and $500 P_{\mathrm{o}}$, respectively (Table 1$)$.

As seen in almost all plots in Figs. 4 and 5, the radiant area of F1 is relatively large and is located in the northern sky. The declination of the geocentric radiants of the particles spans from about $40^{\circ}$ up to $82^{\circ}$. Most radiants have declination between $60^{\circ}$ and $80^{\circ}$. The period of activity of the corresponding shower is predicted to be quite long, up to 41 days, from November 15.8 to December 27.0. A relatively high geocentric velocity, 44 to $46 \mathrm{~km} \mathrm{~s}^{-1}$, implies good conditions for the observation of the shower.

Actually, the real counterpart of the filament F1 can be found in the video data we used. The shower can be identified to the December $\kappa$-Draconids, No. 336 in the IAU MDC list of established showers ${ }^{2}$ (Jopek \& Kaňuchová 2014). The shower was discovered in the video data by the SonotaCo (2009) team and by Jenniskens et al. (2016). The agreement between the predicted

2 https://www.ta3.sk/IAUC22DB/MDC2007/Roje/roje_lista. php?corobic_roje=1\&sort_roje=0 

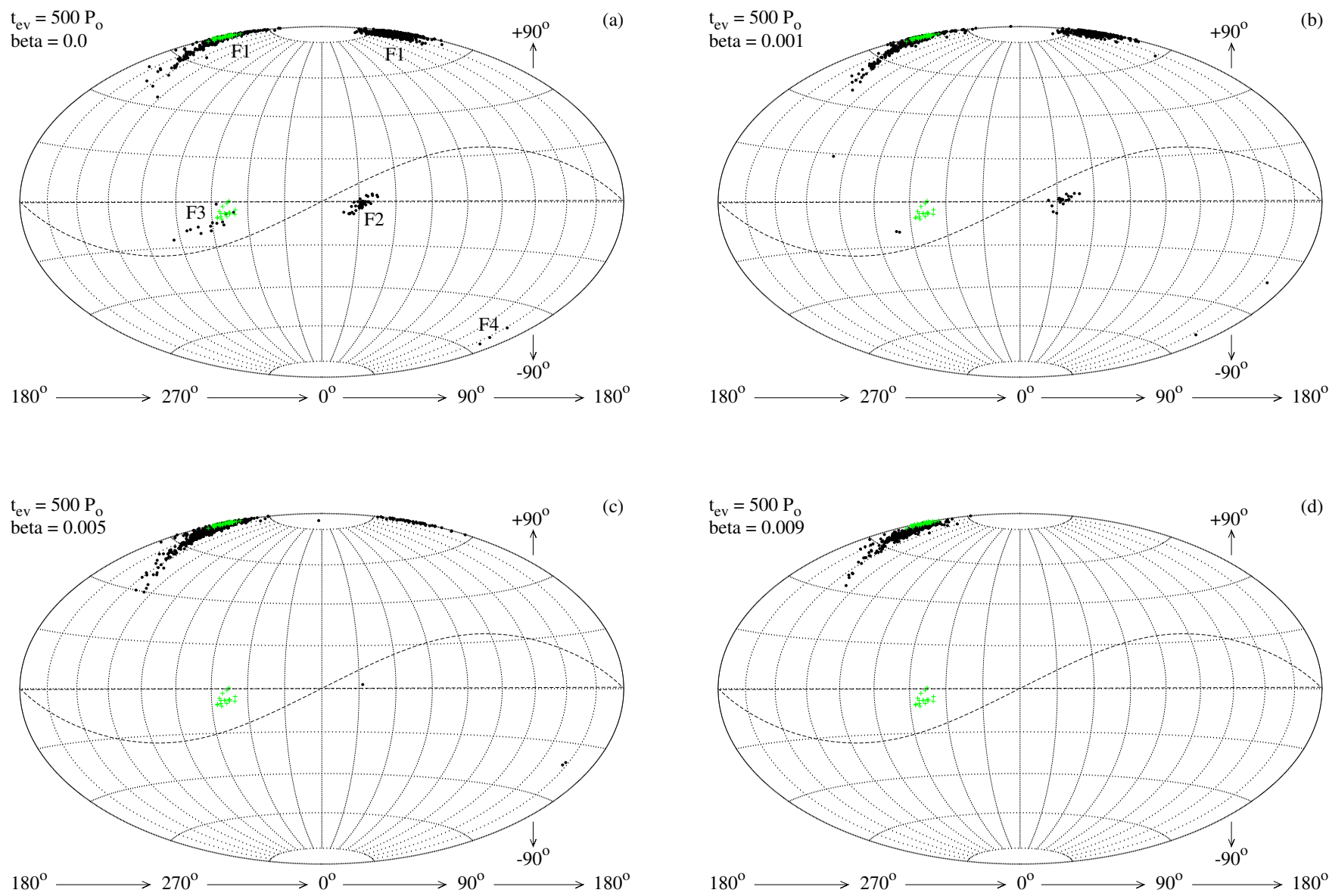

Fig. 4. Distribution of geocentric radiants of modeled particles, which approach the Earth's orbit within 0.05 au at the present. These radiants are indicated by full black circles. The streams were modeled for $t_{\mathrm{ev}}=500 P_{\mathrm{o}}$ and the strength of the P-R drag characterized with value of parameter $\beta=0$ (plot a)); $0.001 \mathbf{~ b}$ ); $0.005 \mathbf{c}$ ); and $0.009 \mathbf{d}$ ). The particles of the 12P-associated stream approach the Earth's orbit in a maximum of four filaments. These are labeled F1, F2, F3, and F4 in plot a). The radiants of the observed video meteors from two corresponding showers are also shown, indicated by green crosses. All radiant positions are shown in the Hammer projection of celestial sphere in the equatorial coordinates. The sinusoid-like curve illustrates the ecliptic.

and actually observed mean characteristics of the shower is good and thus, the identification of F1 to the December $\kappa$-Draconids is reliable enough.

The longitude of the ascending node of mean radiant is not much different from the longitude of the ascending node of the Earth's apex. Because of this fact and the large declination as well as the ecliptical latitude of the mean radiant, the shower obviously contributes to the toroidal meteor structure (a more detailed discussion is presented in Sect. 5).

\subsection{Filaments F2 and F4}

The radiants of the particles constituting filament F2 are located closely to the ecliptic, their mean ecliptical latitude is predicted to be about $-10^{\circ}$. In respect to the equator, the radiants are situated closely below it in the model for $t_{\mathrm{ev}}=350 P_{\mathrm{o}}$ and on both sides of this border of northern and southern celestial hemispheres in the model for $t_{\mathrm{ev}}=500 P_{\mathrm{o}}$. F2 was found in the models for these two evolutionary times. (A single meteor in this filament also appeared in the model for $t_{\mathrm{ev}}=100 P_{\mathrm{o}}$ and $\beta=0$.) It is well predicted, with the help of 43 particles approaching the Earth's orbit within $0.05 \mathrm{au}$, only in the model for $t_{\mathrm{ev}}=500 P_{\mathrm{o}}$.
In the models for $\beta=0$ and both $t_{\mathrm{ev}}=100$ and $350 P_{\mathrm{o}}$, it consists of one and five particles, respectively. Nevertheless, the shower corresponding to $\mathrm{F} 2$ is predicted to have a compact radiant area (Figs. 4a,b; Fig. 5a).

Filament F4 is the poorest filament. Since it is predicted only by three particles, maximum, and in the unreliable model for $t_{\mathrm{ev}}=500 P_{\mathrm{o}}$ whether or not we have anything concrete to say about the predicted filament at all is questionable. If it existed, its period of activity could be predicted to last about three days, from December 4.4 to December 7.6 (Table 2). Of all filaments, $\mathrm{F} 4$ has the lowest declination, less than $-50^{\circ}$, and the highest geocentric velocity, almost $48 \mathrm{~km} \mathrm{~s}^{-1}$ for $\beta=0$ (Table 2).

No real shower corresponding to filament F2 or F4 was noticed in any database used. It is not, however, very surprising since both filaments have few particles and, in particular, the predicted angular distance of mean radiant from the Sun in time of predicted maximum of activity is lower than $75^{\circ}$ for $\mathrm{F} 4$ and lower than $35^{\circ}$ for $\mathrm{F} 2$ (see values of $\gamma$ in the last column of Table 2). Therefore, both predicted showers are the daytime showers. Some meteors of F4 could be seen also in the night sky, but only for a short time before the sunrise. It is difficult to detect the meteors of these two filaments, especially those of F2. 

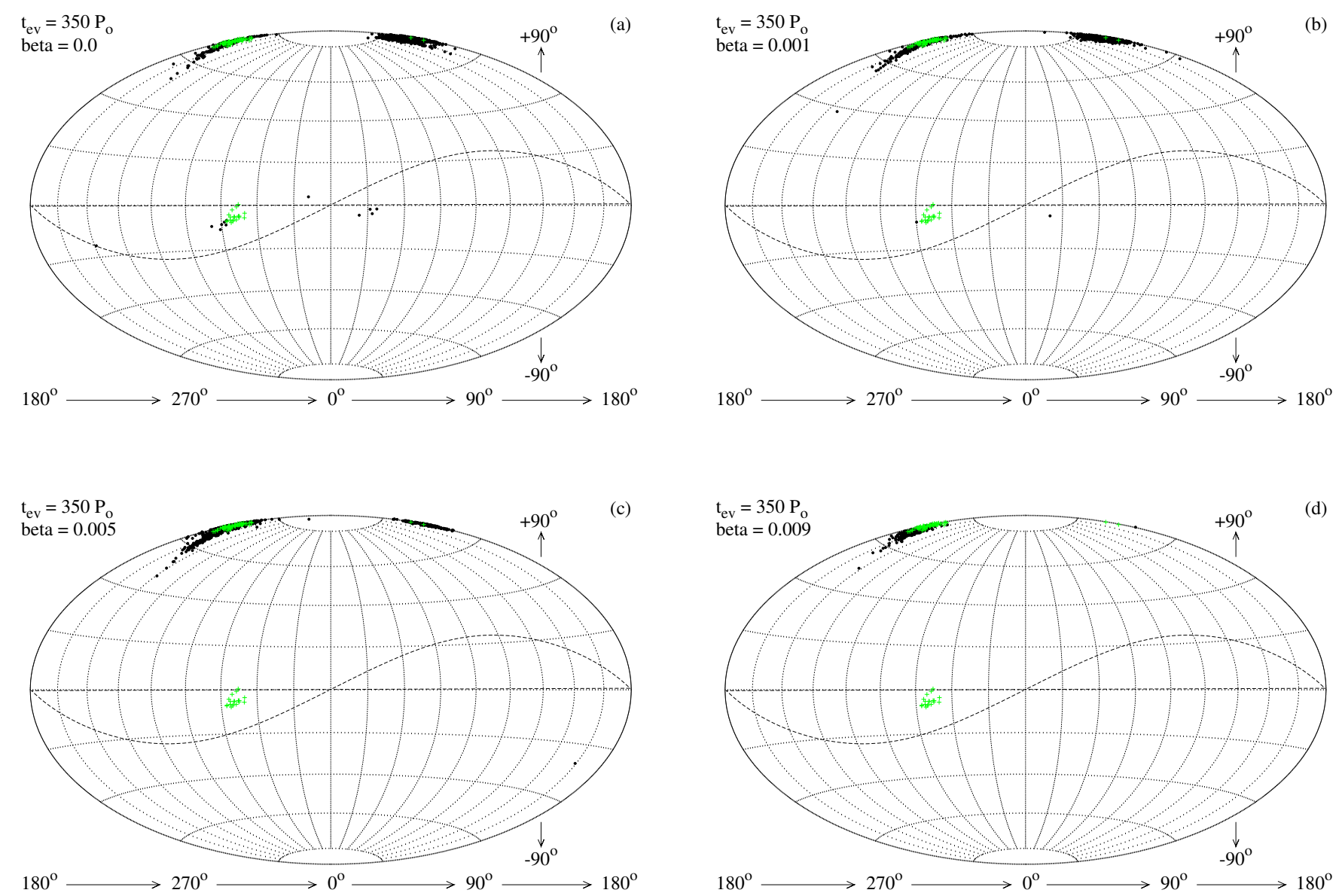

Fig. 5. Distribution of geocentric radiants of modeled particles, which approach the Earth's orbit within 0.05 au at the present. The distribution is shown in the same way as that in Fig. 4, but here for the streams modeled for $t_{\mathrm{ev}}=350 P_{\mathrm{o}}$.

\subsection{Filament F3 and Northern June Aquilids}

When the mean predicted orbit of the filament F3 is considered as the initial orbit in the iteration performed within the breakpoint method of the separation of shower from the SonotaCo as well as EDMOND video databases, the procedure finds a concentration of meteors, which can be regarded as a meteor shower. And, it appears that this shower can be identified to the Northern June Aquilids, No. 164 in the IAU MDC list of established showers. In this list, the shower is reported not only in the video (Holman \& Jenniskens 2012; Jenniskens et al. 2016), but also in the radio-meteor data (Sekanina 1976; Brown et al. 2008). In more detail, Sekanina discovered the shower by the radar system of the Radio Meteor Project at Havana, Illinois. The network was operated from October 1968 to December 1969. Northern June Aquilids were confirmed by the Cameras for All-sky Meteor Surveillance (CAMS) in 2011 (Holman \& Jenniskens 2012). The shower was also recognized from IMO single-station video observations (Molau et al. 2012).

The iteration procedure finds the mean characteristics of the real shower, which are different from the predicted initial orbit. Hence, the predicted and separated showers can be two different showers. A further analysis is necessary. In the studied case of filament F3, some mean orbital as well as geophysical predicted characteristics are nevertheless similar to their counterparts of the Northern June Aquilids. Thus, although an identification of the filament F3 to the Northern June Aquilids is questionable, it is worthwhile to discuss their similarity and diversity.

In the model for $t_{\mathrm{ev}}=100 P_{\mathrm{o}}$, the filament is represented with only a single particle, when $\beta=0$. For a higher values of this parameter, filament does not occur. Besides the model for $t_{\mathrm{ev}}=$ $100 P_{\mathrm{o}}$ and $\beta=0$ and the uncertain modeling for $t_{\mathrm{ev}}=500 P_{\mathrm{o}}$, F3 is predicted by two models for $t_{\mathrm{ev}}=350 P_{\mathrm{o}}$, those for $\beta=0$ and 0.001 . The latter consists of only a single particle, so our comparison between the prediction and data for Northern June Aquilids is done for F3 yielding for the exclusively gravitational dynamics, with $\beta=0$.

In the comparison, we notice a relatively large difference between the high predicted values of mean heliocentric and geocetric velocities and significantly lower observed mean values of these parameters. The difference is then reflected in a large difference between the predicted and observed mean semi-major axes.

Differences of this kind may not necessarily be serious enough to reject the relationship between the predicted and observed shower. For example, Wu \& Williams (1995) revealed the structure of the well-known major shower Perseids with the gaps in the distribution of semi-major axis, whereas the real dispersion of reciprocal semi-major axis ranged from about $0.2 \mathrm{au}^{-1}$ down to zero. Another analysis of the Perseid filamentary structure was done by Kaňuchová et al. (2005) and Svoreň et al. (2006) who found the upper border of the reciprocal semi-major axis of orbits in the shortest filaments equal to about $0.3 \mathrm{au}^{-1}$. 
This value corresponds to the semi-major axis, $a$, not much exceeding $3 \mathrm{au}$. This is actually a small value for the Perseids, which starts in orbits around their parent comet, 109P/SwiftTuttle having $a=26$ au.

In Tables 1 and 2, we notice a vague similarity between the predicted (F3) and observed (Northern June Aquilids) angular orbital elements as well as in the coordinates of mean radiant. However, the semi-major axis of the predicted filament F3 ranges from 14.9 to 18.1 au with a single exception of 31.1 au in contrast to the intervals $(0.93,3.12 \mathrm{au})$ in the SonotaCo data (with one exception of $12.4 \mathrm{au}$ ) and $(1.17,3.23 \mathrm{au})$ in the EDMOND data. The predicted semi-major axis tends to decrease with an increasing P-R strength, but the filament is deflected more and more from the collisional course with the Earth. The decrease is not large enough until the filament disappears completely. The predicted $a$-interval is clearly different from that in the observational video data.

This difference is related to that in mean geocentric velocity. While our predicted $V_{\mathrm{g}}=43.3 \mathrm{~km} \mathrm{~s}^{-1}$ (for $t_{\mathrm{ev}}=350 P_{\mathrm{o}}$ and $\beta=$ 0 ), Brown et al. (2008) from the radio data (CMOR) found the value of $38.4 \mathrm{~km} \mathrm{~s}^{-1}$ and Holman \& Jenniskens (2012) as well as Jenniskens et al. (2016) from the video data (CAMS) determined $V_{\mathrm{g}}=38.3 \mathrm{~km} \mathrm{~s}^{-1}$. An even smaller value, $V_{\mathrm{g}}=36.3 \mathrm{~km} \mathrm{~s}^{-1}$, was found by Sekanina (1976). The larger value, $V_{\mathrm{g}}=43 \mathrm{~km} \mathrm{~s}^{-1}$, approaching to our prediction, was determined by Molau et al. (2012).

The period of activity predicted on the basis of particles in filament F3 in the model for $t_{\mathrm{ev}}=350 P_{\mathrm{o}}$ is from June 16.7 to June 29.2. The model for $t_{\mathrm{ev}}=500 P_{\mathrm{o}}$ naturally indicates a significantly longer period of activity, from May 16.6 to July 10.5 .

Filament F3 is not very abundant in any model and this is another negative circumstance questioning the identification of this filament to the Northern June Aquilids. In conclusion, the question whether this shower is actually related to comet $12 \mathrm{P}$ remains open.

\section{Ecliptic-toroidal structure of the 12P-stream}

In several studies in the past, the stream of a given parent body was predicted to approach the Earth's orbit in several filaments, the radiant areas of which were situated, on the sky, symmetrically to the apex of the Earth's motion (e.g., Neslušan et al. 2013a,b; Neslušan \& Hajduková 2014; Jakubík \& Neslušan 2015). Perhaps the most remarkable are the almost identical complexes of comet 96P/Machholz and asteroid 2003 EH1 (Neslušan et al. 2013a,b), which consist of three pairs of the filaments (four of these filaments were reliably identified to the real showers: daytime Arietids, Northern and Southern $\delta$-Aquarids, and Quadrantids). The filaments of each pair are symmetrical with respect to the Earth's apex.

Four predicted filaments of the stream of 12P also create a structure with the apex symmetry. In Fig. 6, the distribution of the radiants of modeled particles in the model for $t_{\mathrm{ev}}=500 P_{\mathrm{o}}$ and $\beta=0$ is shown in the ecliptical coordinate frame. This frame is, however, modified in one aspect: the ecliptic longitude is shifted about $270^{\circ}$ to place the Earth's apex on the origin of the coordinate system. We can clearly observe the symmetry of filaments F1 and F4, as well as F2 and F3 in respect to the origin of the coordinates, that is in respect to the Earth's apex. It is intriguing that the symmetrical structure occurs, in full, in the uncertain modeling for $t_{\mathrm{ev}}=500 P_{\mathrm{o}}$. Interestingly, a chaotic evolution of the parent and, obviously, the similar orbits of meteoroid particles during a certain period in the far past does not result in a

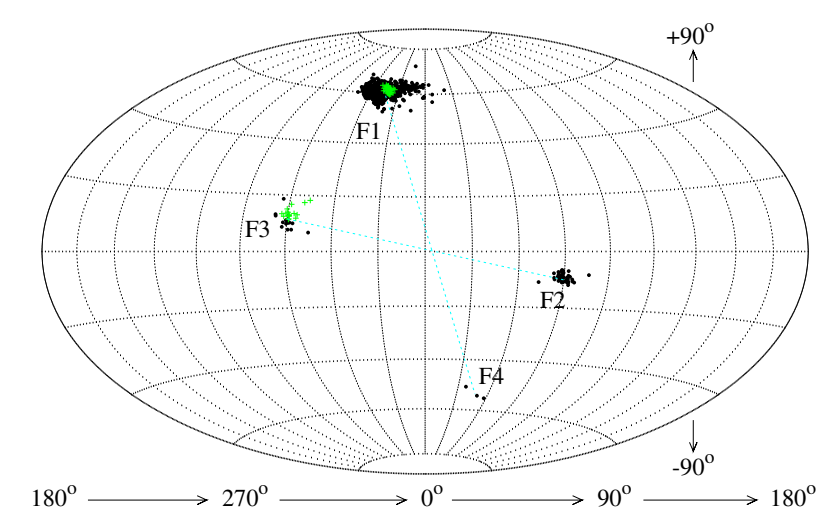

Fig. 6. Distribution of geocentric radiants of modeled particles, which approach the Earth's orbit within 0.05 au at the present in the ecliptical coordinate frame with the modified ecliptic longitude to place the Earth's apex into the coordinate origin. This distribution was derived from the model for $t_{\mathrm{ev}}=500 P_{\mathrm{o}}$ and the strength of the P-R drag characterized with value of parameter $\beta=0$. The radiant positions are shown in the Hammer projection of celestial sphere. The mean radiants of filaments F1 and F4 as well as F2 and F3 are connected (light blue dashed lines) to show the symmetry of the radiant areas.

large dispersion of the stream. It remains situated in well-defined corridors.

The position of radiant areas $\mathrm{F} 1$ and $\mathrm{F} 4$, in a relative vicinity of the poles of ecliptic, classifies these filaments as toroidal. In contrast to F1 and F4, filaments F2 and F3 are situated nearly on the ecliptic, therefore we can classify them as ecliptical.

\section{Conclusions}

The modeling of the stream of meteoroids released from the nucleus of comet 12P/Pons-Brooks and following their dynamical evolution revealed the stream structure. The particles in the stream could approach the Earth's orbit in four discrete filaments. However, only the most abundant of these filaments can be identified to the corresponding real shower, which was separated from the video-meteor data. This is the nighttime shower known as the December $\kappa$-Draconids, No. 336 in the IAU MDC list of established showers. The shower was not predicted for the evolutionary period of $50 P_{\mathrm{o}}(\sim 3500$ years $)$, therefore the lifetime of its meteoroids must be longer.

Of another filaments, it is worthwhile to point out a vague similarity of some mean characteristics of filament F3 to the Northern June Aquilids, No. 164. The predicted geocentric and heliocentric mean velocities are, however, significantly larger than their observed counterparts. Consequently, the interval of the predicted values of semi-major axes is clearly separated from the corresponding interval of the observed $a$-values. Hence, comet $12 \mathrm{P}$ is not, most probably, the parent body of the Northern June Aquilids.

If a shower corresponding to filament F3 existed, it would have be older than the December $\kappa$-Draconids, since F3 occurred only in the model for the evolutionary period $t_{\mathrm{ev}}=350 P_{\mathrm{o}}$ ( 25000 years) and longer.

Two daytime showers are predicted to be even older $\left(t_{\mathrm{ev}} \geq\right.$ 35000 years), if they exist. No similar real shower to any of these predictions was found in the observational data. This could be, however, expected in the case of not-very-numerous daytime showers.

Two nighttime and two daytime predicted showers have their radiant areas symmetrical with respect to the apex of the Earth's motion. The radiant areas of the December $\kappa$-Draconids and their 
daytime counterpart, filament F4, are situated in a vicinity of the north and south poles of the ecliptic and, hence, they can be regarded as toroidal showers of the 12P's complex. On the otherhand side, the radiant areas of both nighttime filament F3 and its daytime counterpart F2 are situated near the ecliptic. So, they can be regarded as the ecliptical showers. The complex of comet $12 \mathrm{P}$ is, thus, another example of the complex with the ecliptictoroidal structure.

Acknowledgements. This research was supported by VEGA - the Slovak Grant Agency for Science (grant No. 2/0031/14). This article was created through realization of the project ITMS No. 26220120009, based on the supporting operational Research and development program financed from the European Regional Development Fund.

\section{References}

Babadzhanov, P. B. \& Obrubov, I. V. 1987, Publications of the Astronomical Institute of the Czechoslovak Academy of Sciences, 67, 141

Babadzhanov, P. B., Williams, I. P., \& Kokhirova, G. I. 2008, MNRAS, 386, 2271

Babadzhanov, P. B., Williams, I. P., \& Kokhirova, G. I. 2013, A\&A, 556, A25

Brown, P., Weryk, R. J., Wong, D. K., \& Jones, J. 2008, Icarus, 195, 317

Chambers, J. E. 1999, MNRAS, 304, 793

Chernitsov, A. M., Baturin, A. P., \& Tamarov, V. A. 1998, Solar System Research, 32, 405

Christou, A. A. \& Vaubaillon, J. 2010, Planet. Space Sci., 58, 1026

Everhart, E. 1985, in Dynamics of Comets: Their Origin and Evolution, Proceedings of IAU Colloq. 83, held in Rome, Italy, June 11-15, 1984, eds. A. Carusi, \& G. B. Valsecchi (Dordrecht: Reidel, Astrophysics and Space Science Library), 115, 185

Hawkins, G. S. 1963, Smithsonian Contributions to Astrophysics, 7, 53

Holman, D., \& Jenniskens, P. 2012, WGN, Journal of the International Meteor Organization, 40, 166

Hunt, J., Williams, I. P., \& Fox, K. 1985, MNRAS, 217, 533

Jakubík, M. \& Neslušan, L. 2015, MNRAS, 453, 1186

Jenniskens, P., \& Vaubaillon, J. 2008, AJ, 136, 725

Jenniskens, P., \& Vaubaillon, J. 2010, AJ, 139, 1822

Jenniskens, P., Nénon, Q., Albers, J., et al. 2016, Icarus, 266, 331

Jones, D. C., Williams, I. P., \& Porubčan, V. 2006, MNRAS, 371, 684

Jopek, T. J., \& Kaňuchová, Z. 2014, Meteoroids 2013, 353

Kaňuchová, Z., \& Neslušan, L. 2007, A\&A, 470, 1123

Kaňuchová, Z., Svoreň, J., \& Neslušan, L. 2005, Contributions of the Astronomical Observatory Skalnate Pleso, 35, 135
Kornoš, L., Koukal, J., Piffl, R., \& Tóth, J. 2014, in Proc. of the International Meteor Conf., eds. M. Gyssens, P. Roggemans, \& P. Zoladek, 23

Lindblad, B. A. 2003, in Solar Variability as an Input to the Earth's Environment, ed. A. Wilson, ESA SP, 535, 755

Lindblad, B. A., Neslušan, L., Porubčan, V., \& Svoreň, J. 2003, Earth Moon and Planets, 93, 249

McIntosh, B. A., \& Jones, J. 1988, MNRAS, 235, 673

Molau, S., Kac, J., Berko, E., et al. 2012, WGN, Journal of the International Meteor Organization, 40, 176

Neslušan, L. 1999, A\&A, 351, 752

Neslušan, L., \& Hajduková, M. 2014, A\&A, 566, A33

Neslušan, L., Svoreň, J., \& Porubčan, V. 1995, Earth Moon and Planets, 68, 427

Neslušan, L., Hajduková, M., \& Jakubík, M. 2013a, A\&A, 560, A47

Neslušan, L., Kaňuchová, Z., \& Tomko, D. 2013b, A\&A, 551, A87

Neslušan, L., Svoreň, J., \& Porubčan, V. 2013c, Earth Moon and Planets, 110, 41

Porubčan, V., Kornoš, L., \& Williams, I. P. 2006, Contributions of the Astronomical Observatory Skalnate Pleso, 36, 103

Sekanina, Z. 1976, Icarus, 27, 265

Sekanina, Z., \& Southworth, R. B. 1975, Physical and dynamical studies of meteors. Meteor-fragmentation and stream-distribution studies, Tech. rep.

SonotaCo. 2009, WGN, Journal of the International Meteor Organization, 37, 55

Southworth, R. B., \& Hawkins, G. S. 1963, Smithsonian Contributions to Astrophysics, 7, 261

Svoreň, J., Kaňuchová, Z., \& Jakubík, M. 2006, Icarus, 183, 115

Sykes, M. V., Greenberg, R., Dermott, S. F., Nicholson, P. D., \& Burns, J. A. 1989, in Asteroids II, eds. R. P. Binzel, T. Gehrels, \& M. S. Matthews, 336

Tomko, D., \& Neslušan, L. 2012, Earth Moon and Planets, 108, 123

Vaubaillon, J. 2004, International Meteor Organization 140, eds. M. Triglavčekada, \& C. Trayner,

Vaubaillon, J., Colas, F., \& Jorda, L. 2005, A\&A, 439, 751

Vaubaillon, J. J., Neslusan, L., Hajdukova, M., et al. 2015, European Planetary Science Congress 2015, held 27 September - 2 October, 2015 in Nantes, France, Online at http: //meetingorganizer . copernicus .org/ EPSC2015/EPSC2015, EPSC2015-423, 10, EPSC2015

Wiegert, P., Vaubaillon, J., \& Campbell-Brown, M. 2009, Icarus, 201, 295

Williams, I. P., \& Ryabova, G. O. 2011, MNRAS, 415, 3914

Williams, I. P., Murray, C. D., \& Hughes, D. W. 1979, MNRAS, 189, 483

Williams, I. P., Ryabova, G. O., Baturin, A. P., \& Chernitsov, A. M. 2004a, Earth Moon and Planets, 95, 11

Williams, I. P., Ryabova, G. O., Baturin, A. P., \& Chernitsov, A. M. 2004b, MNRAS, 355, 1171

Wu, Z., \& Williams, I. P. 1992, in Chaos, Resonance, and Collective Dynamical Phenomena in the Solar System, ed. S. Ferraz-Mello, IAU Symp., 152, 329

Wu, Z, \& Williams, I. P. 1993, MNRAS, 264, 980

Wu, Z., \& Williams, I. P. 1995, MNRAS, 276, 1017 